\title{
Frontières
}

\section{Les médicaments dans le suicide}

\section{Michel Pozo, Brian L. Mishara et Anne Second-Pozo}

Volume 16, numéro 1, automne 2003

Remède ou poison?

URI : https://id.erudit.org/iderudit/1073759ar

DOI : https://doi.org/10.7202/1073759ar

Aller au sommaire du numéro

Éditeur(s)

Université du Québec à Montréal

ISSN

1180-3479 (imprimé)

1916-0976 (numérique)

Découvrir la revue

\section{Citer cet article}

Pozo, M., Mishara, B. L. \& Second-Pozo, A. (2003). Les médicaments dans le suicide. Frontières, 16(1), 37-43. https://doi.org/10.7202/1073759ar

\section{Résumé de l'article}

L'utilisation des médicaments, et notamment des psychotropes, dans la vie courante a entraîné des changements importants dans la problématique du suicide : pour la première fois, un moyen thérapeutique est utilisé pour se donner la mort et la proportion de tentatives non létales pour chaque décès par suicide a augmenté énormément. Les médicaments constituent le troisième mode de suicide au Canada comme en France, et ils sont impliqués dans plus de $80 \%$ des hospitalisations pour tentatives de suicide dans ces deux pays. Les liens entre médicaments et suicide sont complexes. Une des façons de comprendre le choix d'un médicament comme moyen de suicide est l'association entre les effets escomptés d'un décès par suicide et les effets escomptés de l'utilisation des médicaments qui ont pour objectif de s'en prendre à la tête, contenu de la psyché, et d'attaquer les pensées, les tensions, la souffrance en les endormant.
Ce document est protégé par la loi sur le droit d'auteur. L'utilisation des services d’Érudit (y compris la reproduction) est assujettie à sa politique d'utilisation que vous pouvez consulter en ligne.

https://apropos.erudit.org/fr/usagers/politique-dutilisation/ 


\section{Résumé}

L'utilisation des médicaments, et notamment des psychotropes, dans la vie courante a entraîné des changements importants dans la problématique du suicide: pour la première fois, un moyen thérapeutique est utilisé pour se donner la mort et la proportion de tentatives non létales pour chaque décès par suicide a augmenté énormément. Les médicaments constituent le troisième mode de suicide au Canada comme en France, et ils sont impliqués dans plus de $80 \%$ des hospitalisations pour tentatives de suicide dans ces deux pays. Les liens entre médicaments et suicide sont complexes. Une des façons de comprendre le choix d'un médicament comme moyen de suicide est I'association entre les effets escomptés d'un décès par suicide et les effets escomptés de l'utilisation des médicaments qui ont pour objectif de s'en prendre à la tête, contenu de la psyché, et d'attaquer les pensées, les tensions, la souffrance en les endormant.

Mots clés : médicaments - suicide psychotropes - tentatives de suicide souffrance-prévention du suicide.

\section{Abstract}

The development of the use of medications, particularly psychotropic drugs, has resulted in significant changes in suicidal behaviours: for the first time in history, a therapeutic agent has been also a common means of ending life and the proportion of non-lethal suicide attempts for each death by suicide has increased significantly. Medications are the third most common means of suicide in Canada and in France and medications account for $80 \%$ of all hospitalizations for suicide attempts in those countries. The relationship between medications and suicide is complex. One way to understand the association between medications and suicide is to examine the desired outcome of medication use and a suicide. In both instances the object may be understood as a suppression or putting to sleep of unwanted and intolerable thoughts, tensions and suffering.

Key words: medications - suicide psychotropic - suicide attemptssuffering - suicide prevention.

\section{$\begin{array}{lllllllll}\text { R } & \text { E } & \text { C } & \text { H } & \text { E } & \text { R } & \text { C } & \text { H } & \text { E }\end{array}$}

\section{LES MÉDICAMENTS DANS LE SUICIDE}

\author{
Michel Pozo, \\ psychologue, doctorant à l'Université Lyon II, \\ Laboratoire de gérontologie clinique. \\ Brian L. Mishara, Ph.D., \\ professeur, Département de psychologie, UQÀM ; \\ directeur du Centre de recherche et d'intervention \\ sur le suicide et l'euthanasie (CRISE).
}

Anne Second-Pozo,

psychologue clinicienne, thérapeute familiale, Lyon.

Mettre fin à ses jours en s'empoisonnant n'est pas un phénomène nouveau. De la ciguë, immortalisée par Socrate, à la mort aux rats, de la jusquiame, poison cardiaque, au «vert de gris », nombre de candidats à la mort sont passés à l'acte en utilisant ces procédés. Depuis les années 1950, la gamme chimico-végétale a été délaissée au profit des produits nés de la technologie médicale. L'explosion de la fabrication et de la consommation dans la vie courante des médicaments, et particulièrement des psychotropes a entraîné des changements importants dans le domaine de la réflexion sur le suicide: c'est la première fois dans l'Histoire qu'un moyen suicidogène n'est qu'une exagération d'un moyen thérapeutique. Faire du bien ou faire du mal, aspects bénéfiques et maléfiques se confondent.

Qu'en est-il alors du lien entre le double aspect curatif et nocif des médicaments et le suicide ? Est-ce que la prise de certaines médications augmente le risque d'un décès par suicide ou est-ce que le fait de prendre certains médicaments (par exemple, les psychotropes) diminue le risque de suicide ? Quel est l'effet de la disponibilité des médicaments comme facteur de risque suicidaire ? Quel est le rôle des représentations sociales des médicaments dans leur utilisation pour se suicider? Voilà quelques questions que nous abordons dans cet article.

\section{MÉDICAMENTS \\ ET MODES DE SUICIDE}

Les procédés employés par les candidats à la mort, pour leur passage à l'acte, font l'objet de nombreuses analyses. En 1969, l'Organisation mondiale de la santé (OMS) consacre une série d'études, à l'échelle mondiale, aux moyens usités et aux justifications avancées par les désespérés pour se suicider. Il en résulte que ceux-ci se sont tués pour 989 motivations distinctes et selon 83 procédés différents (Monestier, 1995). Nous n'avons pas de données mondiales plus récentes. Cette même série d'études soulignait que les armes à feu, la pendaison et l'empoisonnement représentent 40 à $60 \%$ des moyens utilisés au niveau mondial, en ne tenant compte bien sûr que des pays qui fournissent à l'OMS des informations sur le sujet.

La proportion de suicides attribuables aux médicaments varie suivant les pays, mais Michel Toussignant et Tina Payette, reprenant différentes études, constatent que, sauf pour le Royaume-Uni, il n'y a pas plus de $16 \%$ des suicides directement reliés aux médicaments (Toussignant et Payette, 1997).

Au Canada, la prise d'une surdose de drogues ou de médicaments constitue la troisième méthode de suicide, après la suffocation, principalement par pendaison, et les armes à feu (tableau 1). Elle représente le deuxième moyen utilisé chez les femmes et le troisième chez les hommes (Statistique Canada, 2002). En France, l'intoxication 
TABLEAU 1 - MÉTHODES DE SUICIDE, SELON LE SEXE, POPULATION DE 10 ANS ET PLUS, CANADA, 1998

\begin{tabular}{lccc}
\hline Méthode & Hommes (en \%) & Femmes (en \%) & Total (en \%) \\
\hline Suffocation & 40,0 & 33,9 & 38,8 \\
Empoisonnement (total) & 22,1 & 41,3 & 26,1 \\
$\quad$ Drogues et médicaments & 8,4 & 31,2 & 13,2 \\
$\quad$ Gaz d'échappement & 7,8 & 5,2 & 7,3 \\
$\quad$ Autre monoxyde de carbone & 1,2 & 1,2 & 1,2 \\
$\quad$ Empoisonnements autres & & & \\
$\quad$ et non précisés & & & \\
Armes à feu & 26,2 & 6,6 & 22,1 \\
Saut d'un lieu élevé & 3,9 & 5,8 & 4,3 \\
Noyade & 2,7 & 5,6 & 3,3 \\
Instruments tranchants & 1,6 & 1,4 & 1,6 \\
Autres et non précisés & 3,5 & 5,4 & 3,9 \\
\hline
\end{tabular}

Nota : Les données ayant été arrondies, leur somme peut ne pas correspondre aux totaux indiqués. Source : Statistique Canada, janvier 2002

\section{FIGURE 1 - SUICIDES TOUTES CAUSES ET SUICIDES PAR MÉDICAMENTS SELON LE SEXE ET L'ÂGE - FRANCE 1996}

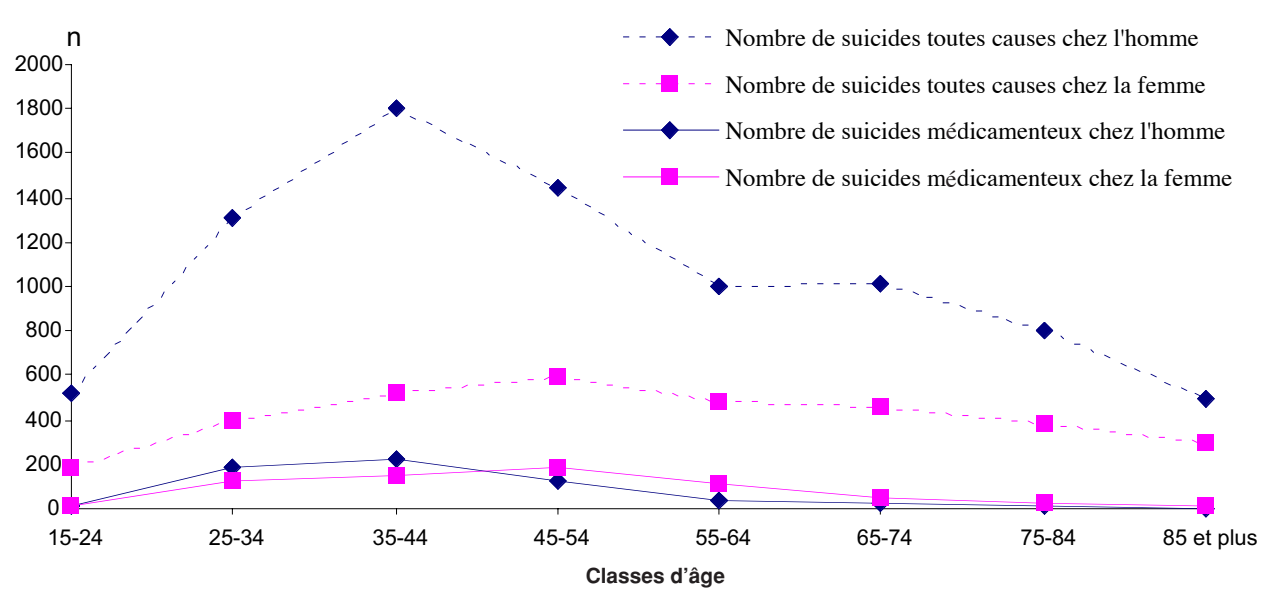

Source: P. SAVIUC et al. (1999) d'après INSERM (1996) : Causes médicales de décès. Résultats définitifs

médicamenteuse, avec $13 \%$ des causes de suicide, arrive également en troisième position, après la pendaison $(37 \%)$ et les armes à feu $(25 \%)$. Cette répartition varie suivant le sexe : c'est le troisième mode de suicide chez l'homme $(8 \%)$, après la pendaison et les armes à feu (ces deux modes étant utilisés dans plus de $70 \%$ des cas). Chez les femmes, trois modes de suicide sont très nettement prédominants: pendaison $(27 \%)$, intoxication volontaire $(25 \%)$ et noyade $(17,5 \%)$, suivie du saut d'un lieu élevé et l'usage d'une arme à feu (respectivement 11 et 7,5\%; Debout, 1998). Ces intoxications sont essentiellement urbaines. Une étude sur Paris et sa banlieue a ainsi montré que les médicaments sont le mode de suicide le plus utilisé à Paris avec 29,9\% des cas. Dans les autres départements d'Île- souvent prescrits aux personnes âgées (Mishara et McKim, 1989). Ainsi, la plupart des personnes âgées au Canada ont à leur disposition des médicaments dont elles peuvent se servir pour mettre fin à leurs jours (Dyck, Mishara et White, 1998).

Pourtant, celles-ci utilisent des moyens plus violents (pendaison, armes à feu) : « un suicide de Viking », disait Malraux à propos du suicide de son grand-père qui se serait fendu le crâne avec une hache. Il est important de souligner qu'il faut probablement y voir un reflet de l'importante sousestimation de ces modes de suicide chez les ânés. En effet, lorsqu'une personne âgée est trouvée morte, s'il n'y a pas d'indications évidentes laissant entrevoir qu'il s'agissait d'un suicide, par exemple l'utilisation d'une méthode observable comme la pendaison ou la présence d'une note d'adieu, on procède très rarement à une enquête approfondie. Dans le cas d'un suicide par médicaments, particulièrement quand l'âné(e) consommait déjà des médicaments pour traiter des problèmes de santé importants, il est rare qu'on effectue des analyses toxicologiques afin de déterminer le rôle des médicaments dans le décès, si l'on ne dispose pas d'autres indications laissant entrevoir la possibilité d'un suicide. On a donc raison de croire que le taux de suicide des aînés par médicaments est significativement en sous-estimation. Cette sous-estimation pourrait en partie expliquer le faible taux de suicide des aînés en général au Québec.

\section{MÉDICAMENTS ET TENTATIVES DE SUICIDE}

Les médicaments sont également impliqués dans une part importante des tentatives de suicide: en 1998-1999, l'empoisonnement était la cause de $83 \%$ des hospitalisations pour tentative de suicide au Canada ( $88 \%$ pour les femmes contre $76 \%$ pour les hommes ; Statistique Canada, 2002), et de $85 \%$ en France $(90 \%$ chez les femmes et près de $80 \%$ chez les hommes; Debout, 2002).

Debout relie d'ailleurs l'apparition des tentatives de suicide à celle des médicaments. En effet, citant Esquirol, sur un plan médical, et Durkheim, sur un plan social, il constate qu'au XIX ${ }^{\mathrm{e}}$ siècle, lorsque ceux-ci se sont intéressés au suicide et à sa compréhension, ils n'envisageaient que la mort par suicide. Ceux qui tentaient de mettre fin à leurs jours n'en réchappaient que rarement, les moyens utilisés étant particulièrement létaux (Debout, 2002).

Ce n'est qu'après la Seconde Guerre mondiale que l'on voit se développer un nouveau type de comportement lié à la diffusion des produits anxiolytiques et des somnifères. Nombre de personnes qui sont tentées d'en absorber au-delà de la dose 
prévue prennent le risque de tomber dans un état comateux avec parfois une complication mortelle. Si la prise médicamenteuse excessive comportait ainsi un risque lourd de complication d'ordre vital, avec les progrès constants de la réanimation, cela semble moins vrai aujourd'hui, la mortalité intrahospitalière étant actuellement faible.

L'absorption de substances médicamenteuses entraîne une phase d'endormissement, puis de coma dans laquelle le corps est progressivement plongé mais qui laisse ouverte la possible intervention des secours (plus ou moins recherchée) qui redonnent vie à ce corps.

La faible gravité somatique des tentatives de suicide médicamenteuses ne doit cependant pas faire négliger l'importance du phénomène, et la gravité potentielle de tout geste suicidaire: une enquête réalisée sous l'égide de l'OMS sur la période 19891992 montrait un taux important de récidive (42\% chez les hommes et $45 \%$ chez les femmes ; Drees, 2001).

Les classes de médicaments en cause sont rapportées dans le tableau ci-dessous et sont principalement représentées par les psychotropes, parmi lesquels les tranquillisants (anxiolytiques et hypnotiques) figurent en bonne place, et les antalgiques / analgésiques. La fréquence des psychotropes, des benzodiazépines et des neuroleptiques est stable. Les antidépresseurs sont en hausse (8\% en 1987, $15 \%$ en 1997 ; Saviuc et al., 1999). Les dérivés du paracétamol (médicaments en vente libre, à visée anti-inflammatoire, antalgique ou antipyrétique) sont concernés une fois sur quatre chez les jeunes (Pommereau, 1996).

L'ingestion des substances est fréquemment polymédicamenteuse : une association de plusieurs toxiques était présente dans environ $45 \%$ des cas de tentatives de suicide rapportées aux centres antipoisons français en 1990-1991.

TABLEAU 2 - CLASSES DE MÉDICAMENTS DANS LES TENTATIVES DE SUICIDES MÉDICAMENTEUSES - ( $\%$ des cas - France)

\begin{tabular}{lccc}
\hline & $\begin{array}{c}\text { Centres } \\
\text { Antipoisons } \\
\mathbf{1 9 9 0 - 1 9 9 1}\end{array}$ & $\begin{array}{c}\text { Bordeaux } \\
\text { Service de réanimation } \\
\text { 1987-1988 }\end{array}$ & $\begin{array}{c}\text { Strasbourg } \\
\text { Services de réanimation } \\
1997\end{array}$ \\
\hline Benzodiazépines & 71,0 & 56,0 & 59,0 \\
Antidépresseurs & 13,0 & 9,5 & 15,0 \\
Tranquillisants & 6,7 & 4,5 & 4,0 \\
Neuroleptiques & 5,6 & 3,9 & 7,0 \\
Barbituriques & 1,0 & 2,5 & 5,0 \\
Analgésiques / Antalgiques & 11,0 & 11,0 & 11,0 \\
Cardio vasculotropes & 7,5 & 2,2 & 30,0 \\
Alcools associés & 14,0 & 25,0 & \\
\hline
\end{tabular}

D'après P. Saviuc et al. (1999)
Dans 20 à $30 \%$ des cas, l'intoxication est associée à une prise d'alcool et/ou à des scarifications à visée phlébotomique (Pommereau, 1998).

On admet en outre que la consommation de toxiques (alcool ou autre) puisse être un facteur important du risque de tentative de suicide. La prise d'alcool accompagne ou précède fréquemment la tentative, quel que soit son mode (toxique ou violent), et la plupart des auteurs soulignent le rôle de l'alcool dans la désinhibition comportementale (Toussignant et Payette, 1997 ; CharazacBrunel, 2002 ; Caillard et al., 1995).

\section{MÉDICAMENTS \\ ET RISQUE SUICIDAIRE} voir que les médicaments soient liés à un risque élevé de suicide: dans un premier temps, on peut se demander si la prise de certains médicaments augmente le risque d'un décès par suicide; dans un deuxième temps, la question qui se pose consiste à savoir si le simple fait d'avoir un accès facile aux médicaments augmente le risque de suicide.

Dans le cas des liens possibles entre la prise des médicaments et un potentiel suicidaire plus élevé, nous avons des difficultés méthodologiques importantes qui rendent une réponse facile à cette question quasiment impossible. Les personnes qui prennent des psychotropes constituent une population plus à risque de se suicider puisque ces psychotropes sont prescrits aux personnes souffrant d'un trouble mental. Un diagnostic de trouble mental constitue en effet le plus important facteur de risque suicidaire (Krug et al., 2002). Il nous est donc difficile de savoir si les personnes qui prennent des psychotropes sont plus à risque de suicide à cause des troubles pour lesquels elles prennent ces médicaments ou
Il existe deux façons dont on peut conce- si la prise de ces médicaments peut avoir pour effet d'augmenter le risque de suicide.

Comment pouvons-nous expliquer une augmentation du risque suicidaire des personnes qui prennent des médicaments qui sont censés être bénéfiques pour elles? Une première explication tient au fait que, malgré les bénéfices des médicaments, il demeure une possibilité d'augmentation du risque suicidaire comme effet secondaire. Jusqu'à présent, on a de façon générale peu d'appuis pour cette hypothèse, bien que des recherches sur certains types de médicaments suggèrent la possibilité d'établir un lien. Une deuxième explication, déjà mentionnée, tient au fait que les personnes qui prennent des médicaments vivent de nombreux problèmes. De plus, le fait d'avoir un trouble psychique entraîne des difficultés interpersonnelles et psychosociales. Par exemple, quelqu'un vivant un trouble psychique présente un risque plus élevé d'avoir un réseau d'appui social moindre que les personnes qui ne souffrent pas de troubles psychiques. L'Enquête Santé Québec a montré que les personnes n'ayant pas de confidents présentaient un risque doublé d'avoir fait une tentative de suicide dans la dernière année (Lavalée et al., 1995). Il est donc possible que toutes sortes de variables liées aux troubles pour lesquels les personnes prennent des médicaments font en sorte que ces personnes ont un risque de suicide plus élevé.

Une troisième explication est que la simple disponibilité des médicaments augmente le risque d'un décès par suicide. En effet, les facteurs culturels d'environnement, d'urbanisation ou géophysiques, l'accessibilité d'un moyen, par exemple, influent notoirement sur les candidats à la mort. Chaque mode comporterait également une part de symbolique porteur de sens: par exemple, on peut émettre l'hypothèse que la pendaison serait la marque de la culpabilité, mais aussi de la quête du rachat, le suicide par coup de feu porterait la marque de l'honneur injustement mis en cause et retrouvé dans la mort. Cela peut être également le feu qui purifie, comme dans les immolations sacrificielles. Quant aux intoxications, elles comporteraient en ellesmêmes cette symbolique de la «mort retrait », chargée de danger, permettant la représentation après la mort d'un corps endormi, qui aurait enfin trouvé le calme et l'apaisement (Debout, 2002). Il existe également des modalités de suicide qui sont plutôt caractéristiques d'une population donnée, comme la crémation chez les Asiatiques, la pendaison ou les armes à feu en milieu rural, l'absorption de produits ménagers corrosifs chez les Maghrébines, l'ingestion de substances agricoles chez les Réunionnais (Pommereau, 1996)... Certains y souscrivent, d'autres s'en démarquent. 
Les représentations sociales associées aux méthodes de suicide jouent un rôle important dans les actes suicidaires. Certaines personnes privilégient une méthode pour mettre fin à leur vie et n'accepteraient jamais de se tuer avec un autre moyen. Le choix d'un moyen peut être basé sur la familiarité avec l'utilisation de ce moyen, l'accès au moyen et notre propre conception de ce qui résultera si un tel moyen est utilisé dans un acte suicidaire. Par exemple, les policiers se suicident habituellement avec leur arme de service en se tirant dans la bouche. Cette pratique fait partie de la culture policière et on peut la comprendre seulement comme une représentation de la façon de mourir «macho » répandue dans leur environnement. Les programmes de prévention du suicide dans le corps policier de la ville de Montréal (Mishara, 2002) ont eu pour effet d'arrêter complètement pendant cinq ans ce type de comportement, soit en moyenne deux suicides par année. Une partie de ce programme de prévention visait à communiquer aux policiers « qu'avaler leur fusil » n'était pas une bonne façon de résoudre leurs problèmes et qu'un tel comportement indiquait un manque de solidarité avec leurs confrères.

L'intérêt porté au mode de suicide utilisé est ancien, notamment pour prévenir la contagion suicidaire. Il y eut des exemples célèbres : à l'hôtel des Invalides, à Paris, il y avait un crochet sous la voûte d'un passage et plusieurs dizaines de personnes s'y pendirent jusqu'à ce qu'on le supprime. Certains sites semblent avoir été conçus comme des lieux pour se suicider. Par exemple, le pont Golden Gate à San Francisco constitue le site où il $\mathrm{y}$ a le plus de suicides parmi tous les sites au monde. Il y a à San Francisco d'autres ponts qui ont un accès aussi facile et qui sont aussi dangereux. Cependant, il est rare qu'on rapporte un suicide sur un autre pont. Le Golden Gate est conçu comme l'endroit où l'on doit se tuer. Dans la ville de Washington, le pont Duke Ellington était connu comme un endroit pour se suicider. À quelques centaines de mètres de ce pont, il y en a un autre aussi élevé. Peut-être à cause de la publicité, on ne rapportait presque jamais de suicide sur l'autre pont, tout le monde utilisait le pont Duke Ellington. À la suite d'une campagne de prévention du suicide, on a construit une barrière pour empêcher les personnes de sauter du pont Duke Ellington, mais on n'a ajouté aucune barrière aux autres ponts de la ville. Cela a complètement éliminé les suicides au pont Duke Ellington et le nombre de chutes des ponts a diminué significativement, sans qu'on note une augmentation des chutes à partir d'autres ponts de la région. De nombreux autres exemples pour-

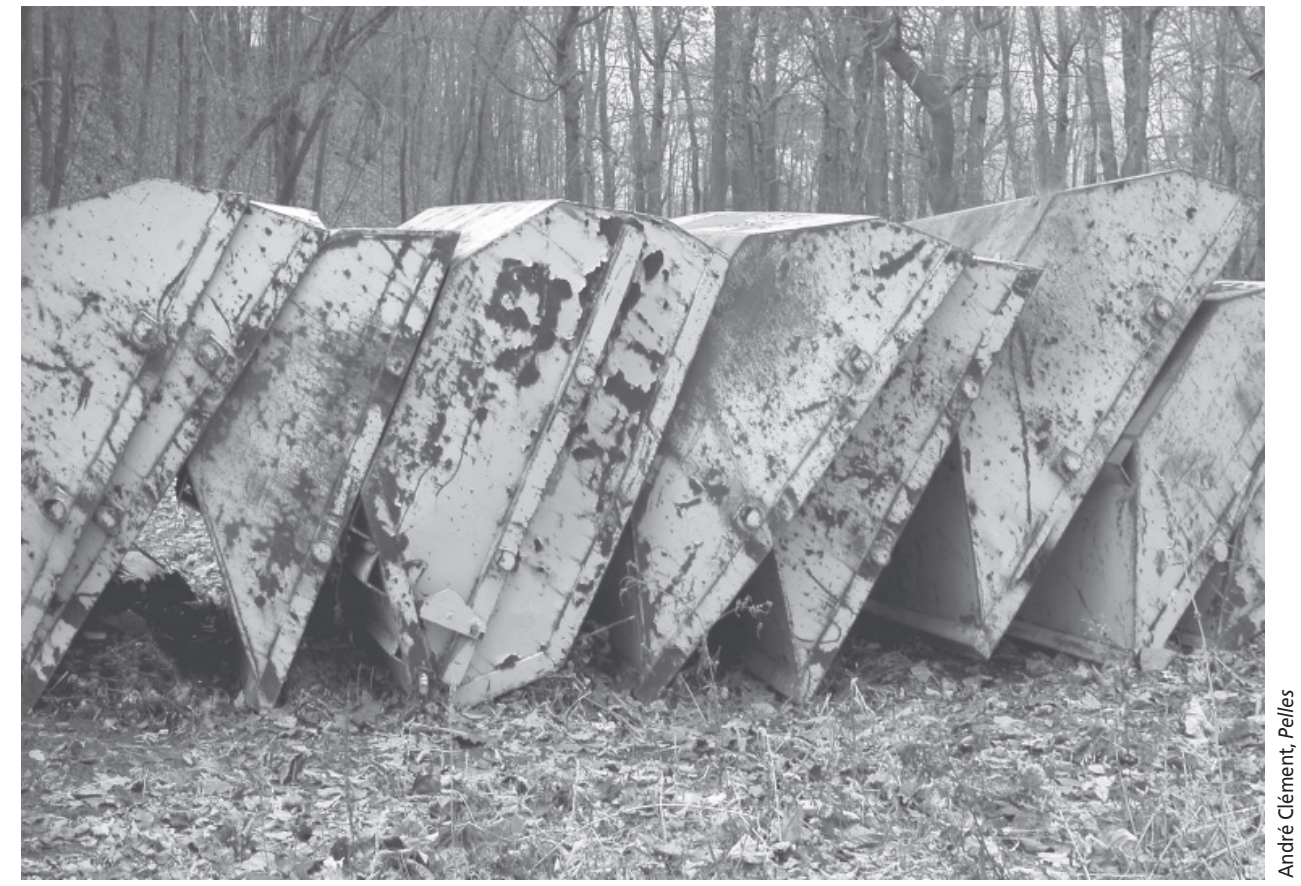

raient êtres cités, notamment sur des lieux ou des monuments au passé dramatique notoire.

Actuellement, certaines études cherchent à évaluer le lien entre l'accessibilité du moyen suicidaire et le risque suicidaire réel. Pour beaucoup, la diffusion élargie au sein de la population générale des médicaments psychotropes ou celle des armes à feu serait responsable de l'augmentation de la fréquence des conduites suicidaires. Dans la mesure où l'impulsivité est une des données fondamentales de l'acte, il est clair que la facilité d'accès à un moyen suicidogène a des conséquences importantes. On utilise ce dont on dispose immédiatement, ce qu'il est facile de se procurer, ce qui n'offre pas de difficultés techniques (Moron, 1999). On semble observer à l'inverse une diminution de mortalité quand des avancées techniques permettent de modifier un moyen suicidogène jusque-là " populaire ». En Australie par exemple, où l'asphyxie par les gaz d'échappement était importante, l'invention du pot catalytique a diminué le nombre de suicides par asphyxie. De même, lorsque le gaz domestique a été détoxiqué en Angleterre, au début des années 1960, le nombre de décès par gaz domestique a chuté et les taux de suicide pour l'ensemble de la population ont diminué proportionnellement (Kreitman et Platt, 1983).

L'extrême banalisation des armes à feu est corrélée avec le risque de mort violente. Contrairement à ce que l'on pourrait croire, la possession d'une telle arme à feu à but défensif accroît le risque de décès, que ce soit par mort violente lors d'une agression ou par suicide. Cela est tout particulièrement vrai chez les jeunes. Aux États-Unis, le taux de suicide est six fois plus important dans les ménages où il y a une arme à feu et les États où la législation facilite la possession d'une arme à feu ont les taux les plus élevés. Globalement, il y a aussi une liaison entre l'évolution des armes à feu depuis 1970 dans la population et le taux de suicide (Philippe, 2000).

Nous pouvons donc conclure que la façon de se tuer qui est favorisée par les personnes suicidaires joue un rôle important dans les actes suicidaires. Lorsqu'un moyen privilégié ou choisi par une personne n'est pas disponible, le risque diminue. La restriction de l'accès aux moyens de suicide apparaît comme une méthode de prévention. Contrairement aux idées reçues, il ne semble pas que la mise à l'écart d'un moyen dangereux ait si peu d'importance que le suicidant cherche forcément une autre manière de mourir. Cela peut être une des explications du taux élevé de suicide dans la population médicale et paramédicale (médecins, vétérinaires, dentistes...) du fait, entre autres, de la facilité d'obtention, voire de la disponibilité permanente des médicaments : le moindre moment anxieux et solitaire coïncidera toujours avec la pleine disponibilité d'une méthode à tout coup mortelle (Caroli et Guedj, 1999).

Guillaume est décrit comme un garçon brillant. À 18 ans, il termine sa première année de médecine et habite avec son frère et ses parents, tous deux pharmaciens, au-dessus de l'officine familiale. Depuis quelque temps, Guillaume semble «avoir décroché », il montre un désintérêt pour ses cours et se replie de plus en plus sur lui-même. 
Ses parents sont encore plus inquiets lorsque leur fils commence à exprimer des idées suicidaires. Ils contactent un médecin psychiatre qui prescrit à Guillaume un traitement antidépresseur. Un samedi, alors que sa famille s'est absentée pour la fin de semaine, Guillaume se retrouve seul au domicile. Il prend les clés de la pharmacie et avale le contenu de deux boîtes d'hypnotiques. Ses parents, ne parvenant pas à joindre leur fils au téléphone, demandent à un ami de se rendre chez eux. Celui-ci trouvera Guillaume allongé dans son lit.

Guillaume est décédé lors du transfert à l'hôpital.

Les médicaments les plus facilement disponibles sont souvent les plus utilisés dans un but suicidaire. La diminution de la prescription des barbituriques est ainsi associée, entre 1973 et 1980, à une diminution significative des morts par surdosage en barbituriques. Dans le même temps, la fréquence des suicides par surdosage en paracétamol ou en benzodiazépines augmentait (Osselton et al., 1984). Les benzodiazépines font partie des médicaments les plus prescrits dans les pays occidentaux, mais c'est en France et en Belgique que la consommation de tels produits atteint des proportions considérables: plus de huit millions de Français absorbent régulièrement des anxiolytiques ; un adulte sur quatre se tranquillise chimiquement (Pommereau et al., 1987). Les psychotropes représentent ainsi en France la deuxième catégorie de médicaments la plus utilisée, après les antalgiques. La banalisation de l'usage des tranquillisants et leur facilité d'obtention conduisant à ce que certains qualifient de «société de l'anxiolyse » où l'intolérance aux frustrations devient déterminante.

Riou et Barriot (1999) rapportent également comment un antipaludéen de synthèse (la chloroquine) a été à l'origine d'intoxications en France. La multiplication des suicides à la chloroquine est expliquée par la facilité d'obtention sans ordonnance de ce médicament, son stockage fréquent dans la pharmacie familiale et par la publication d'un ouvrage « recette» pour réussir son suicide, aujourd'hui interdit. Le Centre antipoison de Paris a ainsi constaté depuis 1982 (date de publication du livre) une augmentation de l'incidence des intoxications graves par la chloroquine. Les trois quarts des personnes ayant ingéré de fortes doses avec des visées suicidaires reconnaissaient avoir lu Suicide mode d'emploi.

Les produits les plus utilisés sont généralement ceux qui sont les plus prescrits, comme nous l'avons souligné. Très souvent, ils l'ont été dans les semaines précédentes, au titre d'une prescription médicale destinée au sujet lui-même ou à un membre de son entourage (Carlsten, Waern et Allebeck, 1999).

Plusieurs études montrent que la prise de médicaments est souvent le fait de personnes dépressives qui suivent un traitement: dans 70 à $80 \%$ des cas, la personne avait absorbé les médicaments que lui avait prescrits son médecin traitant $(51 \%)$ ou son psychiatre (32\%) moins d'un mois avant le geste $(47 \%): 4 / 5$ des suicidants étaient déprimés, 3 / 5 étaient traités par un antidépresseur (Saviuc et al., 1999). Debout parle ainsi des intoxications médicamenteuses comme d'un mode de «suicides dépression », s'inscrivant dans un processus de retrait, d'abandon et de repli. Les modes de «suicides-destruction » (pendaison, arme à feu, arme blanche, précipitation...) comporteraient plutôt une symbolique de refus, de protestation, de violence à ce qui fait violence, entraînant une vision du corps dégradée et brutalisée (Debout, 2002).

Nous disposons depuis peu d'une preuve indiquant que lorsqu'un médicament est rendu moins accessible, cela entraîne un effet en prévention du suicide. En Angleterre, où le paracétamol a été la première cause de décès par empoisonnement, le gouvernement a strictement limité le nombre de comprimés placés dans chaque contenant vendu, et il a aussi limité le nombre de contenants qu'une personne peut acheter dans une même pharmacie la même journée. Avant la mise en place de ces restrictions, il était facile d'acheter un nombre de comprimés potentiellement létal. Hawton (2002) a analysé les décès par paracétamol ainsi que les empoisonnements et les décès par suicide en général. Il a conclu que le contrôle de l'accès au paracétamol en Angleterre a eu pour effet de diminuer le nombre de suicides par paracétamol et cette diminution a été reflétée à la fois par une baisse des décès par suicide faisant appel à toutes les substances toxiques, ainsi que dans les taux de suicide pour le pays. Hawton conclut que le contrôle de l'accès aux médicaments peut avoir un effet de prévention du suicide et qu'il n'y a pas eu d'indication d'un phénomène de substitution par un autre médicament ou une autre méthode.

Comment pouvons-nous comprendre les effets de contrôle d'accès aux médicaments? Les théoriciens expliquent que les dynamiques du suicide mettent souvent l'accent sur le fait qu'un acte suicidaire se produit à un moment où il existe une combinaison de facteurs de risques et où la personne suicidaire vient de subir un « événement déclencheur» (Shneidman, 1985). Un décès par suicide peut donc souvent se produire à un moment précis qu'on appelle une «situation de crise». Si un moyen de se tuer est facilement disponible, il y a risque élevé de passage à l'acte. Par exemple, pour un homme souffrant de dépression, le fait d'apprendre que sa femme l'a quitté peut faire en sorte qu'il est à risque de suicide cette journée-là. Mais si l'accès à un moyen à utiliser n'est pas facile, il est possible que le lendemain ou plusieurs jours plus tard, la personne ait trouvé d'autres façons de se sentir mieux et que la situation de crise aiguë soit déjà chose du passé.

\section{HYPNOS ET THANATOS}

L'une des façons de comprendre le choix d'un médicament comme moyen de suicide est l'association entre les effets escomptés d'un décès par suicide et les effets escomptés de l'utilisation des médicaments. Les somnifères, qui sont fréquemment utilisés dans les tentatives de suicide, ont pour effet de provoquer le sommeil. On associe le sommeil à la mort. En effet, les jeunes enfants ont une conception de la mort comme un état qui ressemble au sommeil (Mishara, 1995). Cette association entre le sommeil et la mort et, pour un grand nombre de sujets, le désir moins de mourir que de tout immobiliser sont fréquemment évoqués, notamment lorsque les modes utilisés sont moins violents. La létalité de la méthode suicidaire employée est évidemment fort différente selon qu'il s'agit d'un moyen violent à effets destructeurs immédiats (pendaison, arme à feu, défenestration...) ou d'une intoxication dont les effets différés et progressifs laissent plus de chances aux secours d'intervenir à temps.

Nous rencontrons Simone, 82 ans, le lendemain de son arrivée à la maison de retraite. Elle avait, quelques jours auparavant, été hospitalisée aux urgences après avoir avalé plusieurs comprimés d'anxiolytiques et de somnifères, le soir du réveillon de Noël, alors qu'elle se trouvait chez son fils et sa belle-fille. Simone exprime une souffrance importante en rapport avec son vécu de tristesse et de solitude depuis le décès de son conjoint. Veuve de son premier mari décédé à la guerre peu avant la libération, elle avait perdu, dix mois auparavant, son deuxième mari avec qui elle se sentait très liée et auprès de qui, elle dit avoir vécu ses plus belles années. Simone se dit isolée chez elle et décrit sa famille, notamment son fils, comme peu présente. Ce vécu de solitude s'est réactivé lorsque son fils et sa belle-fille se sont absentés quelques instants le soir du réveillon pour saluer des voisins. Simone, évoquant son passage à l'acte, raconte qu'elle «ne voulait pas mourir, mais tout arrêter». Simone 
aujourd'hui va bien, les idées de tristesse ne sont plus présentes dans le discours et elle a pu investir un étayage social au sein de l'institution dans laquelle elle vit.

On constate que dans l'intoxication médicamenteuse, les substances employées sont pour la plupart censées induire un profond sommeil. Parallèlement, les dérivés du paracétamol sont connus pour leur action contre la douleur et les maux de tête. Cette léthargie toxique attaque les pensées et les tensions en les endormant. S'en prendre à la tête, cet espace corporel, contenu de la psyché, et marquer un temps d'arrêt, une mise entre parenthèses, sorte d'absence à la vie à un moment donné. L'état de bien-être ainsi recherché par l'utilisation des médicaments utilisés pour le traitement de la dépression peut s'avérer symbolique. Il est cependant possible que le fait de croire que les médicaments vont produire une mort certaine, subite et sans douleur soit la raison pour laquelle on choisit les médicaments. Lorsqu'on entend parler des décès produits par la prise de médicaments, il est rare qu'on ait des indications d'une souffrance prolongée ou l'expérience d'angoisse de la personne pendant sa mort. Les médias et les vedettes d'Hollywood véhiculent des messages et des images qui donnent à penser que les morts par ingestion médicamenteuse sont de «belles morts». La population ignore que très souvent les personnes qui meurent par intoxication souffrent lors du processus.

Il existe cependant encore des situations critiques liées aux propriétés toxiques des molécules ou au terrain affaibli du patient intoxiqué, et ce notamment chez les aînés. Ainsi, il est possible qu'à cause de leur état de santé plus fragile et des effets des divers médicaments qu'ils doivent prendre, il y ait plus de risques qu'une tentative "pas sérieuse » finisse par tuer la personne (Mishara, 1997).

Nous citerons ici l'exemple d'Amélie,

17 ans, hospitalisée aux urgences pour intoxication. Quelques heures plus tôt, elle avait absorbé de l'alcool ainsi qu'une dose importante de somnifères. Les somnifères appartenaient à sa mère. Ses parents étaient partis chez des amis et lui avaient dit qu'ils rentreraient aux environs de 22 heures. Amélie a réalisé son geste vers 20 heures. Elle savait que ses parents rentreraient; elle voulait, selon ses dires, «leur faire peur pour qu'ils fassent attention à elle, leur montrer qu'elle existe ». Par un concours de circonstances, ses parents sont rentrés à minuit. Amélie dira alors, à son arrivée aux urgences, qu'elle ne voulait pas mourir, que ses parents « auraient dû rentrer plus tôt». Quelques instants plus tard, Amélie sombrait dans le coma et il fut impossible de la réanimer.

La mortalité relative aux toxiques psychotropes est inférieure à $1 \%$ alors que pour les toxiques cardiotropes, elle s'élève à près de $4 \%$, ce qui reste nettement inférieur à la mortalité observée avec les pesticides, les

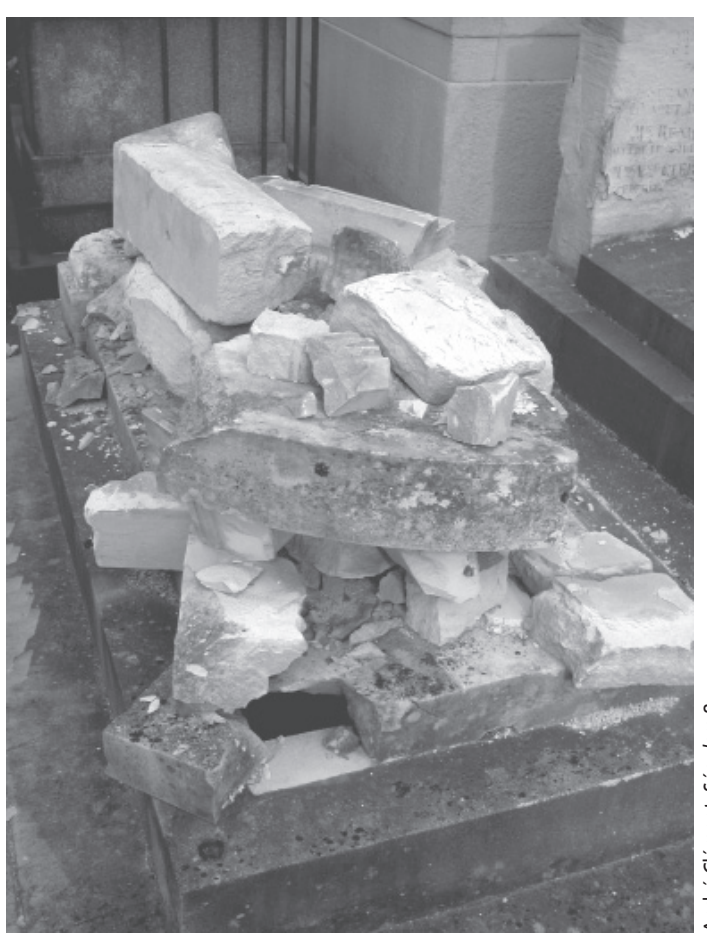

agents de l'environnement et les produits industriels (Hantson, 1999).

\section{MÉDICAMENTS ET PRÉVENTION DU SUICIDE}

On a recours aux médicaments pour traiter les maladies et diminuer la souffrance qui en résulte. Théoriquement, les médicaments psychotropes qui sont utilisés pour diminuer la souffrance psychique devraient avoir un effet de prévention des décès par suicide qui sont l'aboutissement d'une grande souffrance, vécue par la personne suicidaire comme étant interminable et insupportable (Shneidman, 1985). Cependant, l'effet des psychotropes pour diminuer le risque suicidaire est sujet à controverses ; d'un côté, des chercheurs comme Isaacsson (2002) interprètent la diminution des taux de suicide dans les pays scandinaves comme étant due à une augmentation parallèle de l'utilisation des médicaments psychotropes pour traiter la dépression. D'autre part, des chercheurs comme Van Praag (2002), résumant des études scientifiques qui comparent des groupes témoins avec placebo avec un groupe sous modifications de psychotropes, arrivent à la conclusion que le risque de suicide n'est pas moins élevé chez les personnes qui prennent des psychotropes. Il interprète l'absence d'une diminution importante du suicide dans le monde au cours des dernières années comme étant une indication du manque d'effet préventif des psychotropes sur le suicide.

Les liens entre les médicaments et le risque de suicide peuvent être compris de deux façons: tout d'abord, est-ce que les personnes qui prennent des psychotropes pour le traitement des problèmes de santé mentale sont plus à risque de suicide? Il est possible qu'on trouve une corrélation entre prise de médicaments et suicide simplement parce que les troubles pour lesquels les gens prennent les médicaments sont liés au suicide et qu'il n'y a aucune augmentation liée à la prise de médicaments en soi. Cependant, il est aussi possible que les effets secondaires liés à l'utilisation des médicaments puissent augmenter le risque de suicide. En effet, de nombreux médicaments ont des effets secondaires assez troublants pour diminuer la qualité de vie et pour augmenter les souffrances et incapacités d'un individu. Les nausées fréquemment associées à certains médicaments utilisés pour combattre le cancer, la dyskinésie tardive associée à la prise des médicaments pour traiter la schizophrénie sont des exemples d'effets 造 secondaires assez importants qui peuvent constituer des sources de stress majeures. Lorsqu'une personne déjà à risque de suicide vit encore plus de stress ou d'incapacité, cela peut avoir pour effet d'augmenter le risque de passage à l'acte. Le psychiatre Brian Tanney (1992) a émis l'hypothèse que parmi les explications possibles des liens entre trouble mental et suicide, les effets secondaires des traitements de ces troubles par médicaments peuvent avoir un effet iatrogénique. Les effets secondaires des traitements entraîneraient ainsi une augmentation des risques plus considérable que les avantages liés à la prise de ces médicaments.

Même si cet effet iatrogénique peut jouer un rôle dans certains suicides, il est évident que la grande majorité des personnes qui prennent des médicaments en retirent des bénéfices. Cependant, nous n'avons pas de preuves scientifiques convaincantes nous permettant de conclure que la prise de médicaments pour le traitement des troubles psychiques diminue toujours le risque de suicide. Il y a certainement un débat concernant les psychotropes servant au traitement de la dépression. Récemment, des études ont démontré que la clozapine utilisée dans le traitement de la schizophrénie peut avoir 
pour effet de diminuer le risque suicidaire (Meltzer et al., 2003). Parallèlement, le lithium semble prévenir les décès par suicide des personnes souffrant de maniacodépression (Tondo et Baldessarini, 2000).

Le lien entre le double aspect curatif et nocif des médicaments, en particulier des psychotropes, apparaît lorsqu'on se pose la question de la prescription de tels traitements dans le cas de pathologies psychiatriques à haut risque suicidaire. On ne peut s'abstenir de la thérapeutique sous prétexte $\mathrm{du}$ risque, sachant que le risque de suicide est encore plus grand hors traitement. L'idée selon laquelle les psychotropes eux-mêmes poussent au suicide par la levée de l'inhibition psychomotrice liée à la dépression semble abandonnée au profit d'une explication du passage à l'acte par la résistance au traitement et par la dimension d'impulsivité de la personnalité. Selon ces études, il n'y a pas de doute quant à la nécessité des thérapeutiques de la dépression ou de la schizophrénie, à cause des risques considérables liés à l'abstention de toute mesure curative.

$$
* * *
$$

Les médicaments ont énormément d'effets bénéfiques. Cependant, la disponibilité des médicaments et peut-être même l'utilisation de certains peuvent augmenter le risque suicidaire de certaines personnes vulnérables. Le suicide a toujours existé, dans tous les pays et à toutes les époques de l'histoire. Puisque le suicide est un phénomène multi-déterminé et que les taux de suicide sont déterminés par plusieurs facteurs en interaction complexe, nous ne pourrons jamais savoir jusqu'à quel point l'accès aux médicaments explique un plus haut taux de suicide aujourd'hui en comparaison avec certains autres moments dans l'histoire. Cependant, nous avons raison de croire que notre conception des médicaments menant, sinon à une "belle mort », du moins à une mort «acceptable», peut avoir pour effet d'augmenter le risque suicidaire. Nous savons que le contrôle de l'accès aux médicaments et les limitations d'accès à des quantités létales peuvent avoir un effet préventif. Mais ces méthodes de prévention du suicide n'abordent pas les causes du suicide et ne sauraient se substituer aux interventions préventives et thérapeutiques.

\section{Bibliographie}

CAILLARD, V. et al. (dir.) (1995). Dépression: Risque suicidaire et indices de gravité, Paris, Dunod.

CARLSTEN, A., M. WAERN et P. ALLEBECK (1999). "Suicides by drug poisonning among the ederly in Sweden 1969-1996 », Soc. Psychiatr. Epidemiol., n ${ }^{\circ}$ 34, p. 609-614.

CAROLI, F. et M.-J. GUEDJ (1999). Le suicide, Paris, Flammarion.

CHARAZAC-BRUNEL, M. (2002). Prévenir le suicide, Paris, Dunod.

COHEN, H. et D. COHEN (1993). «La surconsommation des psychotropes chez les personnes âgées », Frontières, vol. 6, n 2, p. 10-13.

DEBOUT, M. (1998). "Mourir sa vie», Santé mentale, $\mathrm{n}^{\circ} 27$, p. 17-23.

DEBOUT, M. (2002). La France du suicide, Paris, Stock.

DREES (2001). «Suicides et tentatives de suicide en France, une tentative de cadrage statistique », Études et Résultats, n 109.

DYCK, R.J., B.L. MISHARA et J. WHITE (1998). «Le suicide chez les enfants, les adolescents et les personnes âgées: constatations clés et mesures préconisées ", Forum National sur la santé: les déterminants de la santé, vol. 3: le cadre et les enjeux, Ottawa, Santé Canada, p. 323-390.

HANTSON, P. (1999). "Démarche diagnostique et principes thérapeutiques des intoxications médicamenteuses volontaires », Médecine thérapeutique, vol. 5, nº 1, p. 25-29.

HAWTON, K. (2002). "United Kingdom legislation on pack sizes of analgesics: Background, rationale, and effects on suicide and deliberate self-harm ", Suicide and Life-Threatening Behavior, vol. 32, no 3, p. 223-229.

ISAACSSON, G. et al. (2002). "Données récentes d'études scandinaves. Traiter la dépression: une stratégie efficace de prévention du suicide", Santé mentale au Québec, vol. XXVII, n 2, p. 235-259.

KREITMAN, N. et S. PLATT (1983). «Suicide, unemployment, and domestic gas detoxification», Great Britain J. Epidemiol. Community Health, no 38, p. 1-6.

KRUG, E.G. et al. (dir.) (2002). Rapport mondial sur la violence et la santé, Genève, Organisation mondiale de la santé.

LAVALÉE, C. et al. (dir.) (1995). Aspects sociaux reliés à la santé. Rapport de l'enquête sociale et de santé 1992-1993, vol. 2, Montréal, Santé Québec.

LECOMTE, D. et al. (1995). «Le suicide à Paris et en Île-de-France », Encéphale, n ${ }^{\circ}$, p. 41-49.

MELTZER, H.Y. et al. (2003). «Clozapine treatment for suicidality in schizophrenia : International Suicide Prevention Trial (InterSePT) », Arch. Gen. Psychiatry, vol. $60, \mathrm{n}^{\circ} 1$, p. 82-91.

MISHARA, B.L. (1995). "Conceptions de la mort et du suicide chez l'enfant: recherches empiriques et implications pour la prévention du suicide», P.R.I.S.M.E., vol. 5, $\mathrm{n}^{\circ} 4$, p 499-508.

MISHARA, B.L. (1997). "Les aînés suicidaires: un défi pour la société et les intervenants », Bulletin trimestriel de l'association internationale francophone des aînés, p. 5-8.

MISHARA, B.L. (2002). «Évaluation du programme de prévention du suicide pour les policiers et policières du Service de police de la Ville de Montréal », Ensemble pour la vie, Montréal, CRISE, UQÀM.

MISHARA, B.L. et W.A. MCKIM (1989). Drogues et vieillissement, Boucherville, Gaëtan Morin Éditeur.

MONESTIER, M. (1995). Suicides, histoire, techniques et bizarreries de la mort volontaire, Paris, Le cherche midi.

MORON, P. (1999). Le suicide (septième édition corrigée), Paris, Presses universitaires de France.

OSSELTON, M.D. et al. (1984). «Poisonningasssociated deaths for England and Wales between 1973 and 1980 », Human Toxicol., no 3, p. 201-221.

PHILIPPE, A. (2000). "Épidémiologie des suicides et des tentatives de suicide. Le devenir des tentatives de suicide», dans T. LEMPERIÈRE (dir.), Dépression et suicide, Paris, Masson, p. 3-21.

POMMEREAU, X. (1996). L'adolescent suicidaire, Paris, Dunod.

POMMEREAU, X. (1998). "L'adolescent suicidaire », Santé mentale, no ${ }^{\circ}$, p. 26-30.

POMMEREAU, X. et al. (1987). «Désinhibition comportementale et tentatives de suicide liées aux benzodiazépines ", Psychologie Médicale, vol. 19, n 5, p. 725-727.

RIOU, B. et P. BARRIOT (1999). «Intoxication aiguë par la chloroquine ", Médecine thérapeutique, vol. 5, n 1, p. 37-43.

SAVIUC, P. et al. (1999). "Épidémiologie des intoxications médicamenteuses volontaires ", Médecine thérapeutique, vol. $5, \mathrm{n}^{\circ} 1$, p. $45-48$.

SHNEIDMAN, E. (1985). Definition of Suicide, Northvale, Jason Aronson inc.

STATISTIQUE CANADA (2002). Rapports sur la santé, vol. 13, n 2 , Ottawa.

TANNEY, B. (1992). "Mental disorders, psychiatric patients, and suicide», dans R.W. MARIS et al., Assessment and Prediction of Suicide, New York, The Guilford Press, p. $277-320$

TONDO, L. et R.J. BALDESSARINI (2000). "Reduced suicide risk during lithium maintenance treatment», J. Clin. Psychiatry, vol. 61 (suppl. 9), p. 97-104.

TOUSSIGNANT, M. et T. PAYETTE (1997). Suicide et toxicomanie: deux phénomènes interreliés, Gouvernement du Québec, Comité permanent de lutte contre la toxicomanie.

VAN PRAAG, H.M. (2002). "Why has the antidepressant era not shown a significant drop in suicide rates?», Crisis, vol. 23, p. $77-82$. 